\title{
New Acousto-Optic Regime of Interaction in Media Possessing Strong Elastic Anisotropy
}

\author{
V.B. Voloshinov*, E.A. DJakonov and N.V. Polikarpova \\ Department of Physics, Lomonosov Moscow State University, 119991 Moscow, Russia
}

\begin{abstract}
We present results on theoretical and first experimental investigation of a new regime of acousto-optic interaction existing in acoustically anisotropic medium. We defined the new regime as "semi-collinear" or "mixed interaction" since it combined properties of the traditional non-collinear diffraction and the pure collinear interaction. The peculiar phenomenon was registered in the tellurium dioxide crystal due to the extremely strong elastic anisotropy of the material. Application of a specific cut of the crystal provided observation of the effect in the middle infrared at the optical wavelength $3.39 \mu \mathrm{m}$ and at the acoustic frequencies limited to $300 \mathrm{MHz}$. The observed interaction was characterized by a non-collinear propagation of incident light with respect to acoustic energy flow and simultaneously a collinear propagation of diffracted radiation along the acoustic energy flow. A brief theoretical analysis of the interaction based on wave vector diagrams and two-dimensional coupled wave equations is included in the presentation. Finally, we describe in the paper, the setup and basic details of the carried out acousto-optic experiment.
\end{abstract}

DOI: $10.12693 /$ APhysPolA.127.36

PACS: $78.20 . \mathrm{Hp}$, 42.79.Jq, 43.35.Sx, 43.35+d

\section{Introduction}

The phenomenon of acousto-optic (AO) interaction is widely used in modern optics, optical information processing and laser technology to regulate parameters of optical beams [1-4]. The major advantage of $\mathrm{AO}$ devices consists of a possibility to regulate simultaneously a few parameters of optical signals in real time. For example, quick action of $\mathrm{AO}$ devices is usually limited to a few microseconds [1-4]. It is evident that the extremely wide applications of the diffraction phenomenon at present stimulate research in the field of fundamental acousto-optics.

The acousto-optic interaction is based on the wellknown phenomenon of diffraction of optical beams by phase gratings induced by acoustic waves in transparent to optical radiation media [1-4]. The effect of light diffraction by acoustically induced variations of density and hence refractive index was predicted by Brillouin in 1922. Ten years later Lucas and Biquard as well as Debye and Sears observed the diffraction effect in a liquid medium. In 1935-1936, Raman and Nath theoretically described the diffraction phenomenon in thin acoustic diffraction gratings. Finally, in 1937 Rytov examined Bragg interaction of light and sound in thick gratings $[3,4]$.

It should be underlined that in the thirties, the interaction was examined only in optically isotropic media, i.e., in liquids, glasses and cubic crystals. As for elastic anisotropy of the media, it was neglected. In general, the interaction was analyzed only in the traditional case of transverse, i.e., non-collinear interaction known

\footnotetext{
* corresponding author; e-mail: volosh@phys.msu.ru
}

for close to orthogonal propagation of optic and acoustic beams with respect to each other. A general scheme of the transverse interaction is shown in Fig. 1.

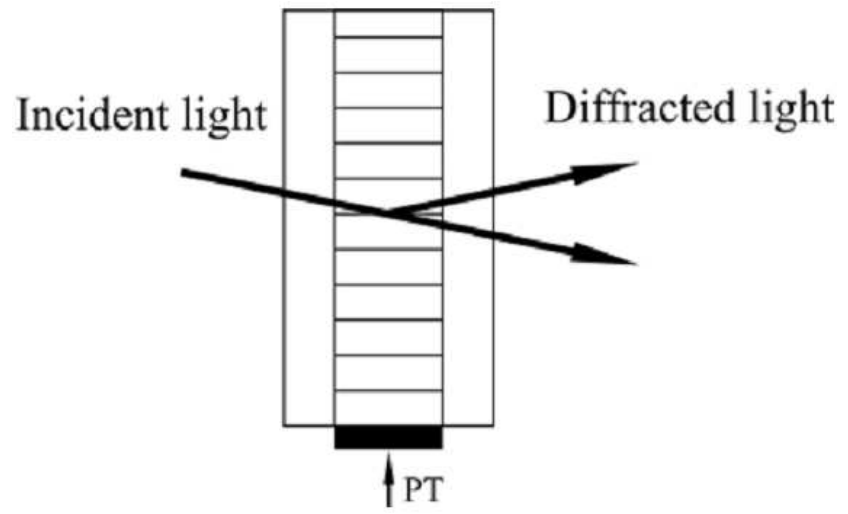

Fig. 1. Transverse scheme of light and sound diffraction.

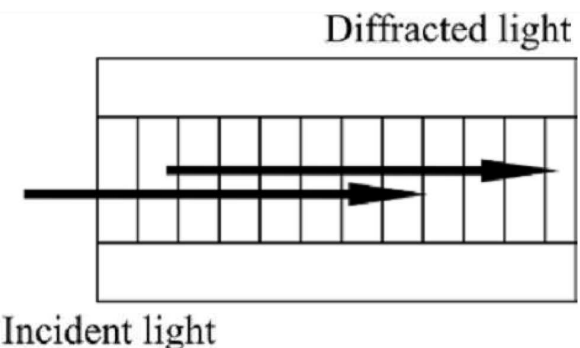

Fig. 2. Collinear scheme of light and sound interaction.

In 1967 Dixon generalized consideration of the diffraction phenomenon over optically anisotropic media. As proved by Dixon [5], not only a transverse but also 
a collinear interaction of optic and acoustic beams may be observed in a birefringent crystal. During the collinear interaction, the incident and diffracted beams propagate collinearly with ultrasound. This interaction is schematically illustrated in Fig. 2. Later a few modifications of the collinear interaction schemes were proposed. The interaction proposed by Kusters with non-collinear phase velocities but collinear group velocities of optic and acoustic beams [6] may be mentioned in this context. The quasicollinear interaction characterized by collinear energy flows of a single optic and acoustic beam is also attributed to the family of the collinear interactions [7].

On the other hand, based on our analysis of the interactions in elastically anisotropic media, we proposed existence of the "semi-collinear" or "mixed" interaction. It was predicted that an incident optical beam could be sent across an acoustic column and therefore propagate approximately orthogonal to acoustic energy flow. As for a corresponding diffracted optical beam, according to expectations, it could propagate strictly collinear with the acoustic energy flow [8-10]. This unusual type of AO interaction is just the subject of the present paper.

\section{Elastically anisotropic media}

It is known that all crystalline materials are elastically anisotropic. It means that in the general case of acoustic propagation, magnitude of phase velocity of ultrasound $V$ depends on direction of the acoustic propagation. The elastic anisotropy also manifests itself in acoustic walkoff angles $\psi$ separating the phase $\boldsymbol{V}$ and group $\boldsymbol{V}_{g}$ velocity vectors of ultrasound [8-12]. Therefore the acoustic columns directed along the Poynting vector are usually tilted relatively to the phase velocity vector, as shown in Fig. 3. It is seen in the figure that the acoustic wave fronts in all three drawings are directed horizontal, while the acoustic energy flow is propagating vertically only in the elastically isotropic case at $\psi=0$.
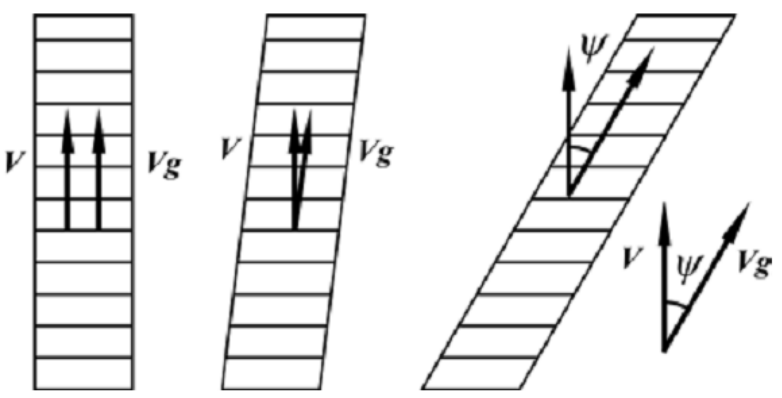

Fig. 3. Acoustic columns in crystals: left drawing illustrates isotropic medium, middle and right drawings correspond to anisotropic media.

As known, there exists a family of crystals demonstrating extremely strong elastic anisotropy. For example, the acoustic walkoff angles in such materials as tellurium dioxide $\left(\mathrm{TeO}_{2}\right)$, calomel $\left(\mathrm{Hg}_{2} \mathrm{Cl}_{2}\right)$, mercury bromide $\left(\mathrm{Hg}_{2} \mathrm{Br}_{2}\right)$, mercury iodide $\left(\mathrm{Hg}_{2} \mathrm{I}_{2}\right)$, etc., may exceed $\psi>70^{\circ}[11-16]$. It means that the conditions of
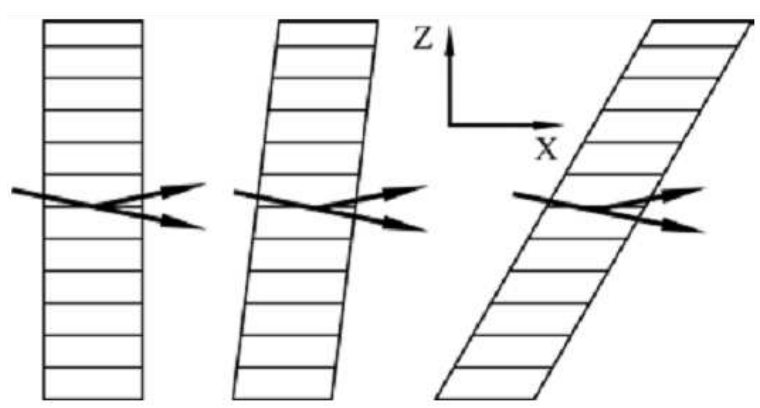

Fig. 4. Propagation of optical beams in elastically isotropic (left drawing) and anisotropic (middle and right drawing) media.

light and sound interaction in the elastically anisotropic materials sufficiently differ from those in the elastically isotropic media [8-10]. This conclusion is illustrated in Fig. 4. As seen, there is no symmetry of the incident and diffracted beams propagation inside the acoustic columns in the elastically anisotropic cases. It means that the phenomenon of light diffraction by ultrasound should be considered and evaluated not only with respect to the axis $x$, i.e., along the acoustic wave front, but also relatively to the axis $z$, i.e., in the direction of acoustic phase velocity vector.

\section{Bragg matching condition in semi-collinear interaction regime}

Existence of the semi-collinear interaction was predicted in Refs. [8-10]. According to conclusions made in the papers, we consider only elastic anisotropy of the interaction medium while optical birefringence is neglected for simplicity. It is known that efficient Bragg interaction of light and sound may take place if phase marching condition is satisfied [1-4]. The matching condition indicates that the wavelength of light $\lambda$, the angle of light incidence $\Theta$, the frequency $f$ and the phase velocity of ultrasound $V$ in an optically isotropic medium are related to each other in the following manner:

$\sin \Theta=\lambda f / 2 n V$,

where $n$ is the refractive index. At fixed wavelength of light $\lambda$, the AO interaction takes place at the frequency of ultrasound

$$
f=2 n V \sin \Theta / \lambda \text {. }
$$

It should be mentioned that Eqs. (1) and (2) are valid in both elastically isotropic and elastically anisotropic media. As for the drawings in Fig. 4 and 5, they illustrate the elastically anisotropic cases of the Bragg interaction.

It is known that in optically isotropic media, the angle of diffraction $\Theta_{d}$ is equal to the Bragg angle of light incidence $\Theta_{d}=\Theta[1-4]$. According to the conclusions of Refs. [8-10], it is possible to direct the diffracted beam along the tilted acoustic column if the angle of light diffraction $\Theta_{d}$ is related to the acoustic walkoff angle in the following manner: 

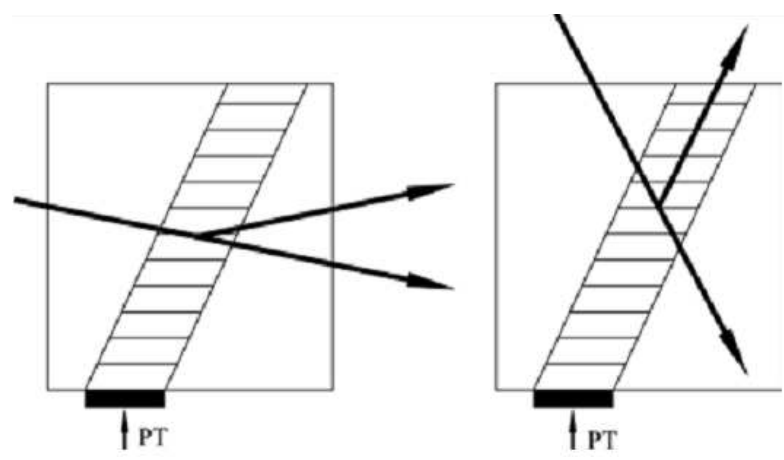

Fig. 5. Mutual directions of interacting beams in case of low (left) and high (right) acoustic frequencies.

$$
\Theta_{d}=90^{0}-\psi
$$

It automatically means that the Bragg angle of light incidence corresponding to the semi-collinear diffraction is equal to

$$
\sin \Theta=\sin \left(90^{\circ}-\psi\right)=\lambda f / 2 n V,
$$

while the acoustic frequency of the Bragg matching is expressed as

$$
f=2 n V \cos \psi / \lambda \text {. }
$$

The right drawing in Fig. 5 illustrates the semi-collinear interaction taking place according to Eq. (5). The left drawing in Fig. 5 shows the diffraction with traditional propagation of beams at the acoustic frequency lower than that in Eq. (5).

\section{Wave vector diagrams of semi-collinear diffraction}

General view of AO cell based on one of the known so far two basic variants of the semi-collinear diffraction is shown in Fig. $6[8,10]$. We consider in the figure the semicollinear diffraction in the particular case of the acoustic walkoff angle $\psi=45^{\circ}$. It is seen in the figure that the transducer launches in the crystal the acoustic wave having energy flow propagating along the axis $z$. The incident light is sent in the cell along the axis $x$ at the Bragg angle $\Theta=45^{\circ}$ with respect to the acoustic wave front. It should be emphasized that the incident light is sent in the cell orthogonally to the acoustic column. In Fig. 6, it intersects the acoustic column horizontally, therefore, the interaction may be defined as the non-collinear or transverse diffraction. As for the diffracted optical beam, it propagates collinearly with the acoustic energy flow along the axis $z$. It means that the interaction is collinear. It is also seen in the figure that the transmitted and the diffracted optical beams propagate in the cell orthogonally with respect to each other.

Wave vector diagram of the semi-collinear interaction is shown in Fig. 7. It illustrates the traditional vector relation

$$
\boldsymbol{k}_{i}+\boldsymbol{K}=\boldsymbol{k}_{\mathrm{d}},
$$

where $\boldsymbol{k}_{i}=2 \pi n / \lambda$ and $\boldsymbol{k}_{\mathrm{d}}=2 \pi n / \lambda$ are the wave vectors of the incident and diffracted light, $\boldsymbol{K}=2 \pi f / V$ is the wave vector of ultrasound. It is seen in Fig. 7 that

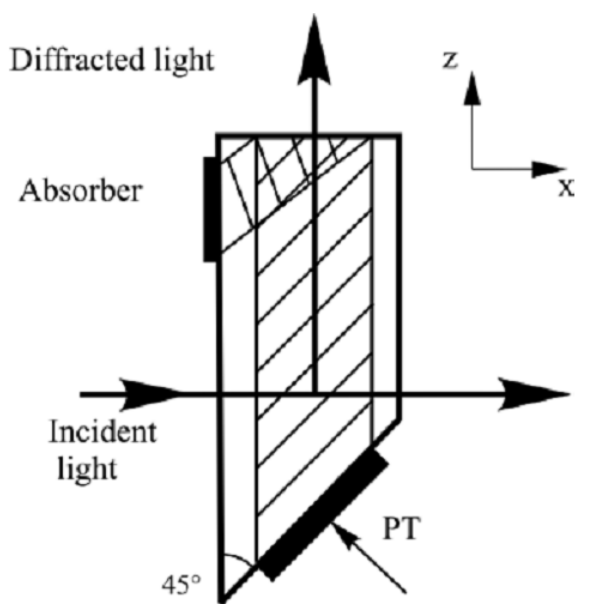

Fig. 6. Acousto-optic cell applying collinear propagation of diffracted light and ultrasound.

the optic wave vectors have equal lengths and they are directed along the axes $z$ and $x$. As usual, the acoustic wave vector connects the ends of the two optic wave vectors. The acoustic vector is directed at the walk off angle $\psi=45^{\circ}$ relatively to the acoustic energy flow.

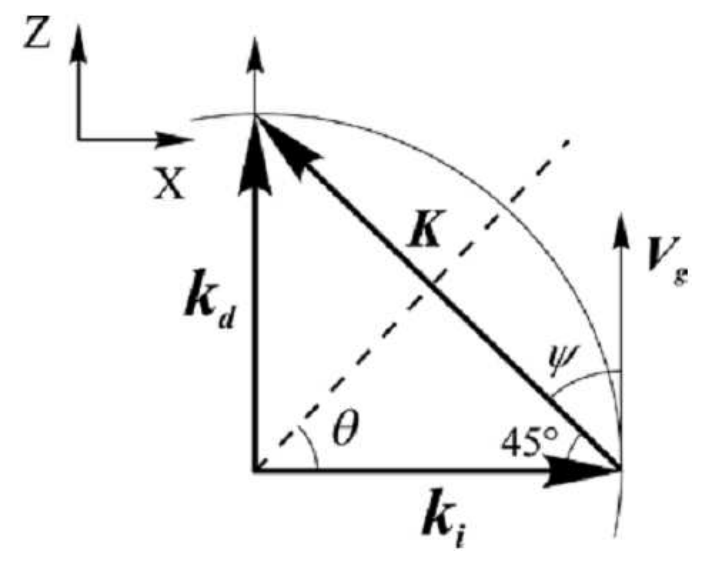

Fig. 7. Wave vector diagram of interaction corresponding to acoustic walk off angle $\psi=45^{\circ}$.

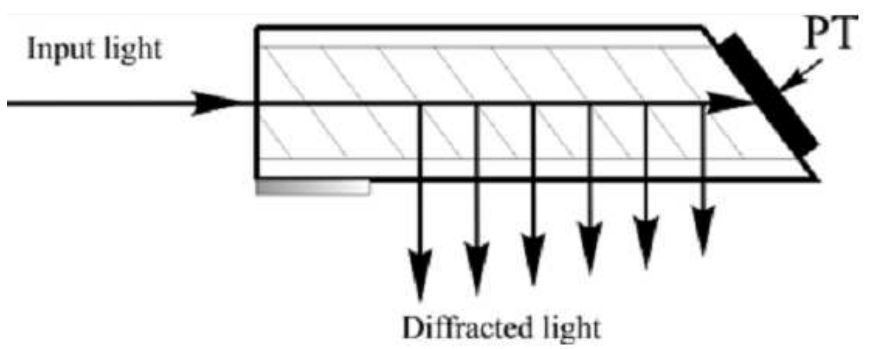

Fig. 8. Acousto-optic cell applying collinear propagation of incident light and ultrasound.

The drawing in Fig. 8 illustrates the second scenario of the semi-collinear interaction. It is a mirror reflection of the semi-collinear interaction shown in Fig. 6. Similarly to the case in Fig. 6, the acoustic walk off angle of the 
medium is equal to $\psi=45^{\circ}$. In the interaction shown in Fig. 8, not the diffracted but the incident optical beam propagates collinearly with ultrasound. However, vector diagram of the interaction, to a great extent, is similar to that in Fig. 7.

\section{Calculation of diffracted light intensity}

The carried out analysis proves that basic parameters of the examined variant of the semi-collinear interaction sufficiently differ from those in the traditional transverse case. It was found that linear aperture of the incident optical beam and height of the acoustic column along the axis $z$ influence on the process of the interaction much stronger than the length of the crystal along the axis $x$. On the other hand, in the second variant of the semicollinear diffraction presented in Fig. 8, the width of the acoustic column is more important as compared to the column length.

It should be underlined that plane wave theory of $\mathrm{AO}$ interaction may not directly be applied to analysis of the semi-collinear diffraction [13-15] because light and sound interaction region is limited not along a single coordinate but along two orthogonal coordinates. It means that the coupled wave equation describing the interaction must be generalized over the two-dimensional case [13-15].

As shown in Sect. 3 of the paper, the semi-collinear diffraction may be observed if the Bragg phase matching condition Eq. (5) is satisfied. Violation of the condition by tuning the acoustic frequency from $f$ to $(f \pm \Delta f)$ results in a decrease of the diffracted light intensity. The violation of the matching condition is evaluated by magnitude of the mismatch vector $\boldsymbol{\Delta} \boldsymbol{k}[3,4]$. As for the diffracted light intensity, it may be found if complex amplitudes of electric fields in the incident, i.e., zero order maximum $C_{0}(x, z)$ and also in the diffracted first order maximum $C_{1}(x, z)$ are known. The total optic field in the crystal $E$ is described by the equation

$$
\begin{aligned}
E & =C_{0}(x, z) \exp \left(\mathrm{i}\left(k_{0 x} x+k_{0 z} z-\omega_{0} t\right)\right) \\
& +C_{1}(x, z) \exp \left(\mathrm{i}\left(k_{1} z-\omega_{1} t\right)\right),
\end{aligned}
$$

where $k_{0 x}$ and $k_{0 z}$ are projections of the incident optic vector $\boldsymbol{k}_{0}$ on the coordinate axes, $\omega_{0}$ and $\omega_{1}$ are cyclic frequencies of light, $\boldsymbol{k}_{1}$ is the wave vector of diffracted light. In this analysis, the angle of light incidence in the zero order of diffraction is defined as $\theta_{0}$, as shown in Fig. 9.

The expression Eq. (7) was inserted in the wave equation in its general form

$$
\nabla^{2} E=\frac{1}{c^{2}} \partial^{2}(\varepsilon E) / \partial t^{2}
$$

where $c$ is the velocity of light in the vacuum and $\varepsilon$ is the dielectric permittivity of a medium. Due to the photoelastic effect, the acoustic wave modulates the dielectric permittivity in the following manner: $\varepsilon=n^{2}+$ $(1 / \pi) q \lambda n \sin (\boldsymbol{K} r-\Omega t)$, where $q$ is the acousto-optic coupling coefficient, $\boldsymbol{r}$ is the radius-vector and $\Omega=2 \pi f$. Using the method of slowly variable amplitudes, we obtained a two-dimensional system of coupled wave equations describing the semi-collinear interaction $[13,15]$ :

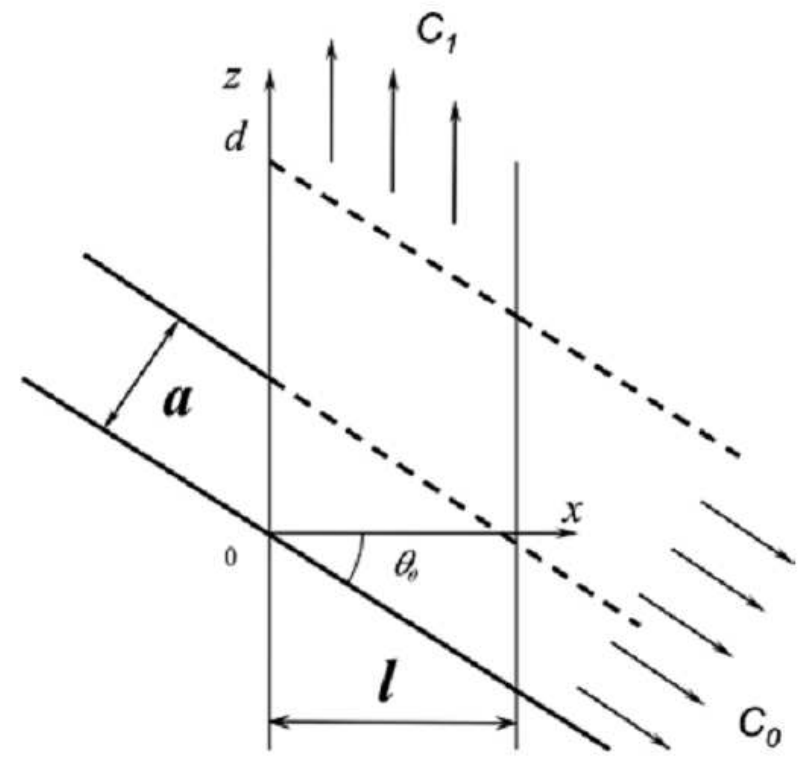

Fig. 9. Directions of optic and acoustic beams in the crystal.

$$
\begin{aligned}
& \frac{\partial C_{0}(x, z)}{\partial x} \cos \theta_{0}+\frac{\partial C_{0}(x, z)}{\partial z} \sin \theta_{0}= \\
& \quad-0.5 q C_{1}(x, z) \exp \left(\mathrm{i}\left(\Delta k_{x} x+\Delta k_{z} z\right)\right) \\
& \frac{\partial C_{1}(x, z)}{\partial z}=0.5 q C_{0}(x, z) \exp \left(-\mathrm{i}\left(\Delta k_{x} x+\Delta k_{z} z\right)\right),
\end{aligned}
$$

where $\Delta k_{x}$ and $\Delta k_{z}$ are the components of the mismatch vector $\boldsymbol{\Delta} \boldsymbol{k}[13,15]$.

Conditions at the border corresponding to the semicollinear interaction are written in the form $C_{0}(0, z)=$ $f(z)$ and $C_{1}(x,-\infty)=0$. The function $f(z)$ determines profile of the incident optic beam over its crosssection. This function satisfies the evident condition $\int_{-\infty}^{\infty}|f(z)|^{2} \cos \theta_{0} \mathrm{~d} z=1$. Then we can find the efficiency of diffraction $I$, i.e., amount of optic energy concentrated in the +1 diffraction order

$$
I=\int_{0}^{l}\left|C_{1}\left(x, z_{\max }\right)\right|^{2} \mathrm{~d} x .
$$

In the above expression, the coordinate $z_{\max }$ indicates limitation on the acoustic column length. As for solution of the coupled wave Eqs. (9) it looks as follows:

$$
\begin{aligned}
& C_{0}(x, z)=f\left(z-x \tan \theta_{0}\right)-0.5 q \int^{\infty} \int_{\tan \theta_{0}}^{\infty} \\
& \left.\quad \times \exp \left(\mathrm{i} \Delta k_{z} g\right) \sqrt{\frac{x g}{g \cos \theta_{0}}}\right) f(z-\zeta) \mathrm{d} \zeta, \\
& C_{1}(x, z)=0.5 q \exp \left(-\mathrm{i}\left(\Delta k_{x} x+\Delta k_{z} z\right)\right) \\
& \quad \times \int_{x \tan \theta_{0}}^{\infty} \exp \left(\mathrm{i} \Delta k_{z} g\right) J_{0}\left(q \sqrt{\frac{x g}{\cos \theta_{0}}}\right) f(z-\zeta) \mathrm{d} \zeta, \quad(11)
\end{aligned}
$$

where $J_{0}$ and $J_{1}$ are the Bessel functions of the first order and $g=\zeta-x \tan \theta_{0}$. Unfortunately, in cases of arbitrary 
function $f(z)$ and strong AO interaction, solution of the system Eq. (9) may be obtained only numerically.

At low diffraction efficiency $I \ll 1$ in the case of an optic beam with a linear aperture $a$ and a uniform distribution of amplitude, it is possible to derive the expression for the diffracted light amplitude in the +1 diffraction order

$$
\begin{aligned}
& \left.C_{1}(x, z)\right|_{z>x \tan \theta_{0}+\frac{a}{\cos \theta_{0}}}=\frac{q a}{2 \cos \theta_{0}} \operatorname{sinc}\left(\frac{\Delta k_{z} a}{2 \cos \theta_{0}}\right) \\
& \quad \times \exp \left(-\frac{\mathrm{i}}{2 \cos \theta_{0}}\left(2 x \left(\Delta k_{x} \cos \theta_{0}\right.\right.\right. \\
& \left.\left.\left.\quad+\Delta k_{z} \sin \theta_{0}\right)+\mathrm{i} \Delta k_{z} a\right)\right)
\end{aligned}
$$

where $\operatorname{sinc}(x)=(\sin x) / x$. In general, the expression $(12)$ provides evaluation of the diffracted light intensity and also of the bandwidth $\Delta f$ of the interaction.

\section{Discussion on results of calculation}

Consideration of the vector diagram in Fig. 7 proves that the mismatch coefficient is equal to $\Delta k_{z}=$ $(2 \pi \Delta f / V) \cos \psi$. It results in the value for the frequency bandwidth

$$
\Delta f=1.8(V / a) \sin \psi \text {. }
$$

Analysis of the expressions (12) and (13) also proves that the diffraction efficiency and the frequency bandwidth are determined by the linear aperture of the incident light $a$ and not by the width of the acoustic column $l$, as it usually takes place in acousto-optics [1315]. It means that the semi-collinear interaction may not be correctly described by one-dimensional coupled wave equation $[1-4,15]$.

Direction of the mismatch vector $\boldsymbol{\Delta} \boldsymbol{k}$ may be found based on the principal conclusion that distribution of optic phase over cross-section of the diffracted beam should not be varying with the coordinate $x[13,15,19]$. In other words, magnitude of the argument of the function $C_{1}(x, z)$ in Eq. (12) does not depend on the coordinate $x$. According to Eq. (12), this requirement is satisfied if $\Delta k_{x} \cos \theta_{0}+\Delta k_{z} \sin \theta_{0}=0$. Therefore, the direction of the mismatch vector is orthogonal not to the acoustic column but to the direction of the incident light propagation. If so, using the vector diagram, one easily determines angular bandwidth of optical scanning $\Delta \theta$ corresponding to the frequency bandwidth of diffraction $\Delta f(13)$ :

$$
\Delta \theta=\frac{\lambda}{2 n V \sin \psi} \Delta f
$$

It is known that during AO interaction, an exchange of energy between an incident optic beam and a diffracted beam takes place in a crystal. In the one-dimensional case, this exchange results in periodic variations of light intensity along a length of interaction. In the twodimensional configuration, this process is more complicated because intensity of interacting beams becomes spatially not uniform $[8,10,13,15]$. For example, the mentioned back transmission of optical energy from the diffracted beam to the zero order beam is accompanied by additional re-radiation of optical energy out of the diffracted beam in the direction of the incident beam. This radiation process is schematically shown in Fig. 9 by arrows adjacent to the incident optic beam. The effect of additional radiation of light was predicted in Refs. [8, 10] and quantitatively confirmed by our calculations $[13,15]$. Data obtained on base of Eq. (11) and presented in Fig. 10 prove this conclusion. The picture shows the theoretically predicted non-uniform distribution of optical energy over section of the incident and diffracted optic beams in $x z$ plane. It should be noted that recent attempts to calculate parameters of the semi-collinear interaction [20, 21] were not successful. The problem originated from a wrong decision to examine a semicollinear device by means of the one-dimensional theory of interaction [20,21].

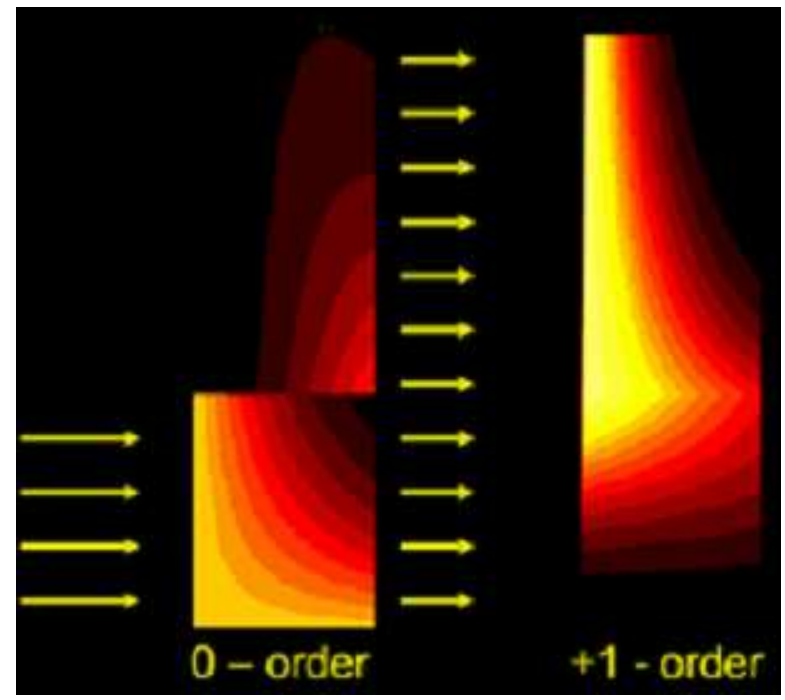

Fig. 10. Distribution of light intensity over crosssection of interaction area.

\section{Selection of material} to observe semi-collinear diffraction

It was found that in the visible light, the semi-collinear diffraction may be observed only at extremely high frequencies of ultrasound. These frequencies belong to the gigahertz frequency domain. For example, the semicollinear diffraction in lithium niobate $\left(\mathrm{LiNbO}_{3}\right)$ crystal at $\lambda=633 \mathrm{~nm}, n_{\mathrm{e}}=2.2, V=3600 \mathrm{~m} / \mathrm{s}$ and $\psi=30^{\circ}[16]$ requires application of the acoustic frequency $f=20 \mathrm{GHz}$. It makes observation of the diffraction problematic because of intolerable attenuation of ultrasound. On the other hand, selection of crystalline materials possessing extremely wide walk off angles $\psi$, low magnitudes of acoustic phase velocity $V$ and application of infrared optic radiation with longer wavelengths $\lambda$ makes it possible to observe the diffraction at suitable acoustic frequencies. For example, in the calomel crystal $\left(\mathrm{Hg}_{2} \mathrm{Cl}_{2}\right)$, at $V=370 \mathrm{~m} / \mathrm{s}, n_{\mathrm{o}}=1.9, \psi=70^{\circ}$ and $\lambda=3.39 \mu \mathrm{m}$, the frequency of the Bragg matching is 
equal to about $f=250 \mathrm{MHz}$. This value is two orders of magnitude lower as compared to lithium niobate. Nevertheless, attenuation of ultrasound in the calomel crystal $340 \mathrm{~dB} /\left(\mathrm{cm} \mathrm{GHz}^{2}\right)$ makes observation of the effect doubtful [17, 18].

Our analysis proved that application of tellurium dioxide crystal occurred quite reasonable as compared to calomel. Selection of a cut of the crystal characterized by the acoustic walk off angle $\psi=72^{\circ}$, the phase velocity $V=720 \mathrm{~m} / \mathrm{s}$ and the index of refraction $n_{\mathrm{o}}=2.3$ required at the optical wavelength $\lambda=3.39 \mu \mathrm{m}$ application of $\mathrm{rf}$ driving signals having the frequencies about $f=300 \mathrm{MHz}$. We evaluate acoustic attenuation in tellurium dioxide at these frequencies as $15-20 \mathrm{~dB} / \mathrm{cm}$ resulting in tolerable lengths of AO interaction.

It should be noted that the examined interaction may be considered as rather unusual if the wavelengths of the optic and acoustic beams are taken into consideration. In the experiment, the magnitudes of the acoustic phase velocity $V=720 \mathrm{~m} / \mathrm{s}$ and the acoustic frequency $f=300 \mathrm{MHz}$ yield the wavelength of ultrasound $\Lambda=V / f=2.4 \mu \mathrm{m}$. On the other hand, the optic wavelength is equal to $\lambda=3.39 \mu \mathrm{m}$. It means that the examined semi-collinear interaction may be defined as the phenomenon of "light diffraction by ultrasound" with some precautions because the optic wavelength is shorter than the acoustic one, i.e., $\lambda>\Lambda$.

\section{Experimental investigation of semi-collinear interaction}

Experimental investigation of the semi-collinear diffraction was carried out in the single crystal paratellurite. We chose the slow shear elastic wave propagating at the angle $\varphi=40^{\circ}$ in (001) plane of the crystal with respect to the axis $x$. Phase velocity of the acoustic wave was equal to $V=716 \mathrm{~m} / \mathrm{s}$ and the acoustic walk off angle equal to $\psi=72^{\circ}$, i.e., close to the maximal value in this crystal $74^{\circ}$. Application of the acoustic wave provided the semi-collinear regime of $\mathrm{AO}$ interaction in the plane (001) at the acoustic frequency $f=300 \mathrm{MHz}$. Moreover, the diffraction was isotropic, i.e., without changes in optic polarization. We used a $\mathrm{He}-\mathrm{Ne}$ laser radiating energy at the wavelength $\lambda=3.39 \mu \mathrm{m}$. The semi-collinear interaction was realized using an extraordinary polarized optical beam. Index of refraction for this beam in the infrared region was equal to $n=2.32$ [22]. The acoustooptic figure of merit in the examined regime of interaction was 3 times as large as compared to that in fused silica and was equal to $M_{2}=4.6 \times 10^{-15} \mathrm{~s}^{3} / \mathrm{kg}$.

Configuration of the examined $\mathrm{AO}$ cell and directions of optic and acoustic waves propagation are schematically shown in Fig. 11. As described in Ref. [12], the slow shear acoustic wave was generated in the crystal due to reflection of a longitudinal wave from the bottom facet of the specimen. Dimensions of the piezoelectric transducer were equal to about $0.1 \times 0.2 \mathrm{~cm}^{2}$. We evaluate the width of the acoustic column in the experiment as $l=0.02 \mathrm{~cm}$ because of spatial compression of the reflected acoustic beam. About $30 \%$ of applied electric power was transformed by the transducer into the acoustic energy of the required acoustic mode.

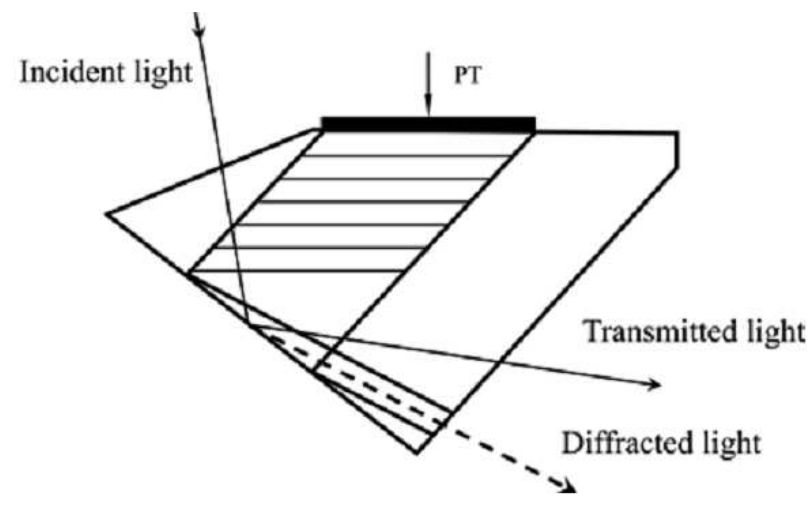

Fig. 11. Configuration of acousto-optic cell and directions of beam propagation.

Special precautions were made to increase sensitivity of registration of optic signals in the used AO setup. The setup included rf sweep generator providing driving signals at frequencies near $f=300 \mathrm{MHz}$. Amplitude of the rf signal was specially modulated at the low frequency $F=700 \mathrm{~Hz}$. Therefore, intensity of the diffracted light was also modulated at this frequency. We used a nitrogen cooled $\mathrm{Ge}: \mathrm{Au}$ detector to register the incident and diffracted radiation. An electronic block terminated to the optical detector was responsible for processing of signals at the low frequency $700 \mathrm{~Hz}$. Amplitude and phase of the signal at output of the block was locked to the driving $\mathrm{rf}$ signal. We also additionally applied a selective voltmeter to detect electric signals at the frequency of modulation $F$. As a result, signal-to-noise ratio in the setup was $50 \mathrm{~dB}$ better as compared to measurements without amplitude and phase locking. It should be emphasized that the electronic part of the setup provided registration of signals with the voltage as low as $1.0 \mu \mathrm{V}$.

The carried out experiments proved that frequency bandwidth of the semi-collinear diffraction was equal to $\Delta f=0.60 \pm 0.04 \mathrm{MHz}$ if an optical beam had the aperture $a=0.2 \mathrm{~cm}$. On the other hand, the bandwidth of the diffraction increased to $1.4 \pm 0.1 \mathrm{MHz}$ in case of a narrower optical aperture $a=0.1 \mathrm{~cm}$. Consequently, we confirmed the general theoretical prediction that the semi-collinear interaction was principally dependent on the linear aperture of the incident optical beam (Eq. (13)).

Experimentally registered magnitude of the diffraction efficiency at $1.0 \mathrm{~W}$ of driving electric signal was equal to $I=3.6 \times 10^{-4}$. It means that $1.0 \mathrm{~W}$ of power of the slow shear wave provided the diffracted light intensity $I=1.2 \times 10^{-3}$. Theoretical prediction at $l=0.02 \mathrm{~cm}$ and $a=0.2 \mathrm{~cm}$ yields $I=1.4 \times 10^{-3}$. We explain the difference between the predicted and measured magnitudes by attenuation of sound. This conclusion is confirmed by data in Ref. [21]. 


\section{Conclusions}

We carried out theoretical and first experimental investigation of the new regime of acousto-optic interaction defined as the semi-collinear interaction. The interaction was observed in the single crystal paratellurite due to the strong elastic anisotropy of the material. The interaction combined properties of the transverse and collinear interactions of light and ultrasound. We described the effect by means of the two-dimensional wave equation. Our theory predicted that the diffraction efficiency and the acoustic frequency bandwidths were dependent on the linear aperture of the incident optical beam rather than on the width of the acoustic column.

It is clear that advantages of the semi-collinear interaction may be recommended for usage, e.g., in notch tunable AO filters. Non-reciprocal devices using the effect seem also of interest to designers of new acousto-optic instruments. Finally, a device based on pulse regime of operation may be used as a source of laser light in which optic energy is radiated orthogonally to borders of acoustic columns while diffracted laser beams are moving along the columns.

\section{Acknowledgments}

The support of the Grant N14-12-00380 by Russian Scientific Fund is greatly appreciated.

\section{References}

[1] J. Xu, R. Stroud, Acousto-optic Devices: Principles, Design, and Applications, Wiley, New York 1992.

[2] A. Goutzoulis, D. Paper, Design and Fabrication of Acousto-optic Devices, Marcel Dekker, New York 1994.

[3] V.I. Balakshy, V.N. Parygin, L.E. Chirkov, Physical Principles of Acousto-optics, Radio and Svyaz Publ., Moscow 1985 (in Russian).

[4] A. Korpel, Acoustooptics, Marcel Dekker, New York 1988.

[5] R.W. Dixon, J. Quant. Electron. QE-3, 85 (1967).
[6] J.A. Kusters, D.A. Wilson, D.L. Hammond, J. Opt. Soc. Am. 64, 434 (1974).

[7] V.B. Voloshinov, Opt. Eng. 31, 2089 (1992).

[8] V.B. Voloshinov, O.Yu. Makarov, Proc. SPIE 3581, 108 (1998).

[9] V.B. Voloshinov, O.Yu. Makarov, in: Proc. World Congress on Ultrasonics, Paris, Acoust. Soc. France, Paris 2003, p. 1.

[10] V.B. Voloshinov, O.Yu. Makarov, Photon. Optoelectron. 5, 53 (1998).

[11] V.B. Voloshinov, AIP Conf. Proc. 1433, 94 (2012).

[12] E.A. Djakonov, V.B. Voloshinov, N.V. Polikarpova, Acoust. Phys. 58, 107 (2012).

[13] E.A. Djakonov, V.B. Voloshinov, in: Proc. 15th Int. School-Seminar "Coherent Optics and Optical Spectroscopy", Kazan State University, Kazan (Russia) 2011, p. 62 (in Russian).

[14] E.A. Djakonov, in: Proc. 13th Russian National School-Seminar "Wave Phenomena in Inhomogeneous Media", Moscow State University, Moscow 2012 (in Russian).

[15] E.A. Djakonov, V.B. Voloshinov, J. Commun. Technol. Electron. 59, 456 (2014).

[16] Acoustic Crystals, Ed. M.P. Shaskolskaya, Nauka Publ., Moscow 1982 (in Russian).

[17] P. Tournois, Opt. Commun. 281, 4054 (2008).

[18] R. Maksimenka, P. Tournois, Opt. Commun. 285, 715 (2012).

[19] A.S. Zadorin, Radiophys. Quant. Electron. 25, 1055 (1982).

[20] E.A. Djakonov, V.B. Voloshinov, N.V. Polikaropova, in: Proc. 14th School-Seminar "Physics and Applications of Microwaves", Moscow State University, Moscow 2013 (in Russian).

[21] E.A. Djakonov, in: Proc. 14th Russian National School-Seminar "Wave Phenomena in Inhomogeneous Media", Moscow State University, Moscow 2014, Sect. 6, p. 6 (in Russian).

[22] N. Uchida, Phys. Rev. B 4, 3736 (1971). 